\section{Atenção oferecida aos idosos portadores de hipertensão: Pesquisa Nacional de Saúde, 2013}

\section{Attention to elderly individuals with hypertension: the Brazilian National Health Survey, 2013}

\section{Atención ofrecida a los ancianos portadores de hipertensión: Encuesta Nacional de Salud, 2013}

\author{
Rosália Garcia Neves 1 \\ Suele Manjourany Silva Duro 1 \\ Thaynã Ramos Flores 1 \\ Bruno Pereira Nunes 1 \\ Caroline dos Santos Costa 1 \\ Andrea Wendt 1 \\ Fernando César Wehrmeister 1 \\ Elaine Tomasi 1
}

\section{Resumo}

O objetivo foi avaliar a atenção oferecida por profissional de saúde aos idosos com hipertensão e as desigualdades, utilizando a escolaridade como indicador socioeconômico, no Brasil. Estudo transversal, com dados da Pesquisa Nacional de Saúde, em 2013. A atenção oferecida foi avaliada por meio dos indicadores: recomendações para alimentação saudável, manter o peso adequado, ingerir menos sal, praticar atividade física, não fumar, não ingerir bebida alcoólica em excesso, fazer acompanhamento regular e solicitação de exames de sangue e urina, eletrocardiograma e teste de esforço. As recomendações para ingerir menos sal (91\%), alimentação saudável (88\%) e fazer acompanhamento regular (88\%) foram mais prevalentes. Idosos com ensino superior completo apresentaram maiores prevalências de recomendações para manter uma alimentação saudável $(R P=1,12)$, manter o peso adequado $(R P=1,21)$, praticar atividade física $(R P=1,29)$, acompanhamento regular $(R P=1,11)$, solicitação do teste de esforço $(R P=2,26)$ e recebimento de todos indicadores $(R P=2,14)$ em relação aos idosos sem instrução. A atenção oferecida por profissional de saúde está deficiente em todo o país. Além disso, as desigualdades ainda persistem. Tendo em vista o aumento da população idosa e das doenças crônicas não transmissíveis, são necessários esforços para melhorar a qualidade da atenção e diminuir as desigualdades.

Doença Crônica; Hipertensão; Qualidade da Assistência à Saúde; Idoso doi: 10.1590/0102-311X00189915

\section{Correspondência}

R. G. Neves

Rua Santa Cruz 1740, apto. 411, Pelotas, RS 96015-710, Brasil. rosaliagarcianeves@gmail.com

1 Universidade Federal de Pelotas, Pelotas, Brasil. 


\section{Introdução}

As doenças crônicas não transmissíveis (DCNT) são as principais fontes de carga de doença no mundo, sendo, no Brasil, responsáveis por cerca de $72 \%$ das mortes 1 . Tais agravos atingem particularmente a população idosa, por conta do processo natural de envelhecimento, e se mostram associados a fatores comportamentais, como atividade física insuficiente, uso de álcool e tabaco e alimentação inadequada 1,2. A hipertensão arterial sistêmica (HAS) é uma condição crônica que afeta aproximadamente 55\% dos idosos brasileiros 3, acarretando hospitalizações, complicações cardiovasculares, cerebrovasculares e renais 1. Essa doença é multifatorial, e os distintos modelos causais reconhecem que, além dos mecanismos biológicos que a influenciam, as determinações sociais podem gerar o acúmulo de padrões de vida que refletem as iniquidades em saúde 4,5.

No Brasil, mesmo com as melhorias evidenciadas na atenção a essa doença, que podem ser atribuídas, em parte, à implantação da Estratégia Saúde da Família (ESF), desigualdades sociais ainda persistem 1,6,7. As desigualdades são diferenças, variações e disparidades encontradas na saúde de indivíduos e grupos populacionais, são evitáveis e passíveis de intervenções políticas, por meio de ações que objetivem a melhoria dos determinantes sociais de saúde 8 . Muitos autores 3,8,9,10,11 vêm estudando as desigualdades em saúde utilizando, como exposição, indicadores de nível socioeconômico tais como: renda, escolaridade e índice de bens.

O Ministério da Saúde preconiza o cuidado integral, resolutivo e de qualidade mediante políticas que objetivam ações focalizadas nas DCNT e não somente voltadas para o modelo de cuidado médico tradicional, mas também com uma perspectiva de prevenção e promoção da saúde 5 . Os portadores de HAS devem ter contato frequente com os serviços e os profissionais de saúde, buscando acompanhamento de sua condição e evitando agravos gerados por essa doença 2 . Sendo assim, barreiras no acesso e baixa qualidade da atenção podem comprometer o desempenho funcional do indivíduo e a sua qualidade de vida além de contribuir para o aumento da carga de doença relacionada 12,13.

Diante desse cenário, o mapeamento das desigualdades em saúde e a identificação de ações capazes de reduzi-las são estratégias importantes para a promoção da equidade. Ainda, a literatura aponta para uma melhoria nos indicadores de cobertura da atenção básica, porém há uma escassez de informações sobre a atenção oferecida pelos profissionais de saúde aos idosos com hipertensão. Nesse sentido, o presente estudo poderá suprir lacunas existentes sobre a temática, bem como embasar as políticas públicas voltadas para essa população, visto que é uma demanda crescente no país. O objetivo foi avaliar a atenção oferecida por profissional de saúde aos idosos com hipertensão e as desigualdades, utilizando a escolaridade como indicador socioeconômico, no Brasil.

\section{Métodos}

Estudo transversal, de base populacional, utilizando dados da Pesquisa Nacional de Saúde (PNS) realizada em 2013, pelo Instituto Brasileiro de Geografia e Estatística (IBGE) em parceria com o Ministério da Saúde. Sua amostra é representativa de moradores de domicílios permanentes, localizados em área urbana ou rural de municípios das cinco grandes regiões geográficas, distribuídos nas 26 Unidades da Federação (UF) e no Distrito Federal.

O processo de amostragem foi feito em três estágios. Primeiramente, foram selecionados os setores censitários, seguido dos domicílios e, por último, os indivíduos com 18 anos ou mais. A amostra da PNS 2013 foi composta por 64.348 domicílios em que 60.202 indivíduos responderam ao questionário sobre doenças crônicas. Desses, 23.815 tinham 60 anos ou mais.

A coleta de dados foi feita por entrevistadores treinados que utilizaram computadores de mão (personal digital assistance - PDA) para armazenamento dos dados. O questionário da PNS foi constituído de três partes: inicialmente, foram coletadas variáveis do domicílio. A segunda parte referiu-se às características gerais de todos os moradores do domicílio, incluindo escolaridade, trabalho, rendimento, deficiências, cobertura de plano de saúde, utilização dos serviços de saúde, saúde do idoso, cobertura de mamografia e características de crianças menores de dois anos de idade. Na terceira parte, questões sobre outras características de trabalho e apoio social, percepção do estado de saúde, acidentes e violências, estilos de vida, doenças crônicas, saúde da mulher, atendimento pré-natal, saú- 
de bucal e atendimento médico foram direcionadas a um morador adulto (18 anos ou mais de idade), selecionado aleatoriamente. Essa seção do questionário só poderia ser respondida pelo morador selecionado, sendo os dados daqueles com 60 anos ou mais utilizados no presente estudo. Mais detalhes sobre o processo de amostragem os instrumentos estão disponíveis no artigo metodológico da PNS 14.

Para avaliar a atenção oferecida, foram utilizadas como indicadores as recomendações para alimentação saudável, manter o peso adequado, ingerir menos sal, praticar atividade física, não fumar, não ingerir de bebida alcoólica em excesso e fazer acompanhamento regular, operacionalizados pela questão: "em algum dos atendimentos para hipertensão, algum médico ou outro profissional de saúde lhe deu alguma dessas recomendações?". Também foram utilizados como indicadores de atenção oferecida por profissional de saúde a solicitação de exames de sangue e urina, eletrocardiograma e teste de esforço, obtidos pela pergunta: "em algum dos atendimentos para hipertensão arterial foi pedido algum exame?”. Além disso, foi criada uma variável dicotômica (sim/não) que avaliou o recebimento de todos os indicadores utilizados, aqui considerados de processo, pois correspondem à prestação da assistência dos serviços e dos profissionais de saúde 15,16. As informações sobre os indicadores foram respondidas somente pelos idosos (indivíduos com 60 anos ou mais) portadores de HAS.

A principal variável de exposição foi a escolaridade (sem instrução, fundamental incompleto, fundamental completo/médio incompleto, médio completo/superior incompleto, superior completo). As variáveis utilizadas para ajuste foram sexo (masculino, feminino); idade em anos completos (60-69, 70-79, 80 ou mais); cor da pele (branca, preta, parda, amarela/indígena); situação conjugal (sem companheiro, com companheiro) e região de residência do entrevistado (Norte, Nordeste, Centro-oeste, Sudeste, Sul).

Todas as análises foram realizadas no pacote estatístico Stata 12.1 (StataCorp LP, College Station, Estados Unidos), utilizando o comando svy para considerar o desenho amostral. Inicialmente, foi realizada uma descrição da amostra segundo as variáveis sociodemográficas e região de residência. Ainda foram obtidas as prevalências e intervalos de $95 \%$ de confiança (IC95\%), e estimadas as razões de prevalências (RP) brutas e ajustadas de cada indicador e do recebimento de todos os indicadores, segundo a exposição principal, por meio de regressão de Poisson 17.

O projeto foi submetido à Comissão Nacional de Ética em Pesquisa e aprovado com protocolo no 10853812.7.0000.0008. Todos os participantes assinaram o Termo de Consentimento Livre e Esclarecido, resguardando os princípios éticos.

\section{Resultados}

Dos 23.815 idosos entrevistados, 5.524 indivíduos referiram diagnóstico de HAS. Desses, 4.974 possuíam dados para os indicadores de atenção oferecida por profissional de saúde utilizados, representando a amostra do presente estudo.

Em relação às características dos indivíduos incluídos, $62,6 \%$ eram do sexo feminino, 52,6\% tinham entre 60 e 69 anos, 53,9\% referiram cor de pele branca e 56,5\% viviam com companheiro. A maior parte tinha o Ensino Fundamental incompleto $(41,6 \%)$ e residia na Região Sudeste $(52,9 \%)$ (Tabela 1).

A Figura 1 apresenta as prevalências de cada um dos indicadores estudados. Entre as recomendações, as mais relatadas foram ingerir menos sal (91,3\%), alimentação saudável (88,3\%), fazer acompanhamento regular $(87,7 \%)$ e manter o peso adequado (83,1\%); as menos referidas foram praticar atividade física (78\%), não fumar $(73,5 \%)$ e não beber em excesso (71,7\%). Em relação à solicitação de exames, sangue e urina foram os mais frequentes, $83,8 \%$ e $73,9 \%$, respectivamente, enquanto o eletrocardiograma $(70,9 \%)$ e o teste de esforço foram os menos solicitados $(37,1 \%)$.

$\mathrm{Na}$ análise bruta, com exceção da recomendação para ingerir menos sal, todos os indicadores de qualidade estiveram associados à escolaridade $(\mathrm{p}<0,05)$. As maiores prevalências da recomendação para alimentação saudável, manter o peso adequado, praticar atividade física, fazer acompanhamento regular e solicitação de teste de esforço foram encontradas entre idosos com Ensino Superior completo (Tabela 2).

Após o ajuste, observou-se que os indicadores recomendação para não fumar e solicitação de exame de sangue perderam a significância estatística. Idosos com ensino superior completo apresentaram 


\section{Tabela 1}

Descrição da amostra de acordo com características sociodemográficas e região de residência em idosos com hipertensão arterial sistólica. Brasil, 2013 ( $N=4.974)$.

\begin{tabular}{|c|c|}
\hline Variáveis & $\begin{array}{c}\text { Amostra total } \\
\text { n (\%)* }\end{array}$ \\
\hline \multicolumn{2}{|l|}{ Sexo } \\
\hline Masculino & $1.689(37,4)$ \\
\hline Feminino & $3.285(62,6)$ \\
\hline \multicolumn{2}{|l|}{ Idade (anos) } \\
\hline $60-69$ & $2.621(52,6)$ \\
\hline $70-79$ & $1.634(32,9)$ \\
\hline 80 ou mais & $719(14,5)$ \\
\hline \multicolumn{2}{|l|}{ Cor da pele } \\
\hline Branca & $2.345(53,9)$ \\
\hline Preta & $511(9,9)$ \\
\hline Parda & $2.054(34,9)$ \\
\hline Amarela/Indígena & $63(1,3)$ \\
\hline \multicolumn{2}{|l|}{ Situação conjugal } \\
\hline Sem companheiro & $2.774(43,5)$ \\
\hline Com companheiro & $2.200(56,5)$ \\
\hline \multicolumn{2}{|l|}{ Escolaridade } \\
\hline Sem instrução & $1.772(31,7)$ \\
\hline Fundamental incompleto & $1.834(41,6)$ \\
\hline Fundamental completo/Médio incompleto & $420(7,6)$ \\
\hline Médio completo/Superior incompleto & $547(10,9)$ \\
\hline Superior completo & $401(8,2)$ \\
\hline \multicolumn{2}{|l|}{ Região de residência } \\
\hline Norte & $682(4,5)$ \\
\hline Nordeste & $1.422(21,4)$ \\
\hline Centro-oeste & $589(6,3)$ \\
\hline Sudeste & $1.530(52,9)$ \\
\hline Sul & $751(14,9)$ \\
\hline
\end{tabular}

* Número absoluto e proporção ponderada da amostra.

maiores prevalências de recebimento de recomendações para manter uma alimentação saudável $(\mathrm{RP}=$ 1,12; IC95\%: 1,05-1,20), manter o peso adequado (RP = 1,21; IC95\%: 1,13-1,29), praticar atividade física (RP = 1,29; IC95\%: 1,20-1,40), para realização de acompanhamento regular (RP = 1,11; IC95\%: 1,05-1,17) e para a solicitação do teste de esforço (RP = 2,26; IC95\%: 1,80-2,83). Idosos com Ensino Fundamental ou Médio incompleto apresentaram uma prevalência de recebimento de recomendação de não ingerir bebidas alcoólicas em excesso 13\% maior (IC95\%: 1,03-1,23), quando comparados àqueles sem instrução. Os idosos com Ensino Médio completo ou Superior incompleto mostraram uma frequência 13\% maior (IC95\%: 1,02-1,25) de solicitação de exame de urina e 32\% maior (IC95\%: 1,21-1,43) de solicitação de eletrocardiograma, quando comparados aos idosos sem instrução. Essas associações foram estatisticamente significativas $(\mathrm{p}<0,05)$ (Tabela 2).

A prevalência de idosos que receberam todos os indicadores de atenção oferecida por profissional de saúde foi 24,2\% (IC95\%: 21,9-26,5). A Tabela 2 também apresenta as razões de prevalências brutas e ajustadas do recebimento de todos os indicadores de acordo com a escolaridade. Na análise bruta, os idosos com Ensino Superior completo apresentaram uma prevalência 2,36 vezes maior (IC95\%: 1,77-3,16) de recebimento de todos os indicadores em relação aos idosos sem instrução. Mesmo após o ajuste, a associação se manteve significativa, porém a medida de efeito reduziu para 2,14 (IC95\%: 1,59-2,86). 


\section{Figura 1}

Prevalências dos indicadores de atenção oferecida por profissional de saúde aos idosos com hipertensão arterial sistólica. Brasil, $2013(\mathrm{~N}=4.974)$.

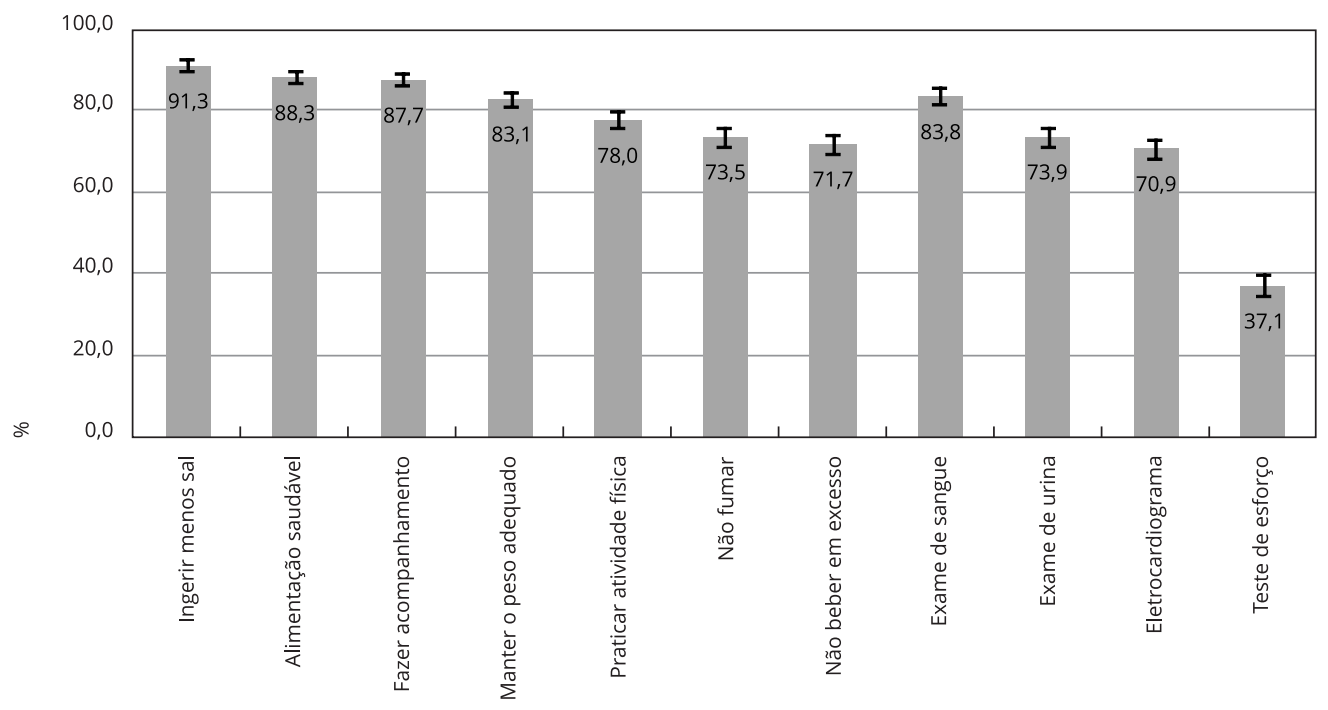

Tabela 2

Prevalência, análise bruta e ajustada dos indicadores de atenção oferecida por profissional de saúde, segundo escolaridade, aos idosos portadores de hipertensão arterial sistólica. Brasil, $2013(\mathrm{~N}=4.974)$.

\begin{tabular}{|c|c|c|c|c|c|c|}
\hline \multirow[t]{2}{*}{ Variáveis } & \multicolumn{5}{|c|}{ Escolaridade [\% (IC95\%)] } & \multirow[t]{2}{*}{ Valor de $p$ * } \\
\hline & Sem instrução & $\begin{array}{c}\text { Ensino } \\
\text { Fundamental } \\
\text { incompleto }\end{array}$ & $\begin{array}{c}\text { Ensino } \\
\text { Fundamental } \\
\text { completo/Médio } \\
\text { incompleto }\end{array}$ & $\begin{array}{l}\text { Ensino Médio } \\
\text { completo/ } \\
\text { Superior } \\
\text { incompleto }\end{array}$ & $\begin{array}{c}\text { Ensino } \\
\text { Superior } \\
\text { completo }\end{array}$ & \\
\hline \multicolumn{7}{|c|}{$\begin{array}{l}\text { Recomendação para alimentação } \\
\text { saudável }\end{array}$} \\
\hline Prevalência & $\begin{array}{c}85,0 \\
(82,2-87,7)\end{array}$ & $\begin{array}{c}87,9 \\
(85,5-90,3)\end{array}$ & $\begin{array}{c}93,2 \\
(90,5-95,8)\end{array}$ & $\begin{array}{c}91,8 \\
(87,9-95,6)\end{array}$ & $\begin{array}{c}93,9 \\
(89,3-98,5)\end{array}$ & \\
\hline Análise bruta & 1,00 & $\begin{array}{c}1,03 \\
(0,99-1,08)\end{array}$ & $\begin{array}{c}1,10 \\
(1,05-1,14)\end{array}$ & $\begin{array}{c}1,08 \\
(1,03-1,14)\end{array}$ & $\begin{array}{c}1,11 \\
(1,04-1,17)\end{array}$ & $<0,001$ \\
\hline Análise ajustada & 1,00 & $\begin{array}{c}1,04 \\
(0,99-1,08)\end{array}$ & $\begin{array}{c}1,10 \\
(1,05-1,15)\end{array}$ & $\begin{array}{c}1,09 \\
(1,03-1,15)\end{array}$ & $\begin{array}{c}1,12 \\
(1,05-1,20)\end{array}$ & $<0,001$ \\
\hline \multicolumn{7}{|c|}{$\begin{array}{l}\text { Recomendação para manter o peso } \\
\text { adequado }\end{array}$} \\
\hline Prevalência & $\begin{array}{c}78,4 \\
(74,9-81,9)\end{array}$ & $\begin{array}{c}81,7 \\
(78,6-84,7)\end{array}$ & $\begin{array}{c}89,7 \\
(86,3-93,1)\end{array}$ & $\begin{array}{c}88,9 \\
(84,7-93,0)\end{array}$ & $\begin{array}{c}93,9 \\
(90,4-97,5)\end{array}$ & \\
\hline Análise bruta & 1,00 & $\begin{array}{c}1,04 \\
(0,98-1,10)\end{array}$ & $\begin{array}{c}1,14 \\
(1,08-1,21)\end{array}$ & $\begin{array}{c}1,13 \\
(1,06-1,21)\end{array}$ & $\begin{array}{c}1,20 \\
(1,13-1,27)\end{array}$ & $<0,001$ \\
\hline Análise ajustada & 1,00 & $\begin{array}{c}1,04 \\
(0,98-1,11)\end{array}$ & $\begin{array}{c}1,14 \\
(1,08-1,21)\end{array}$ & $\begin{array}{c}1,13 \\
(1,06-1,21)\end{array}$ & $\begin{array}{c}1,21 \\
(1,13-1,29)\end{array}$ & $<0,001$ \\
\hline
\end{tabular}

(continua) 


\begin{tabular}{|c|c|c|c|c|c|c|}
\hline \multirow[t]{2}{*}{ Variáveis } & \multicolumn{5}{|c|}{ Escolaridade $[\%$ (IC95\%)] } & \multirow[t]{2}{*}{ Valor de $p$ * } \\
\hline & Sem instrução & $\begin{array}{l}\text { Ensino } \\
\text { Fundamental } \\
\text { incompleto }\end{array}$ & $\begin{array}{c}\text { Ensino } \\
\text { Fundamental } \\
\text { completo/Médio } \\
\text { incompleto }\end{array}$ & $\begin{array}{l}\text { Ensino Médio } \\
\text { completo/ } \\
\text { Superior } \\
\text { incompleto }\end{array}$ & $\begin{array}{l}\text { Ensino } \\
\text { Superior } \\
\text { completo }\end{array}$ & \\
\hline \multicolumn{7}{|c|}{ Recomendação para ingerir menos sal } \\
\hline Prevalência & $\begin{array}{c}90,7 \\
(88,4-93,0)\end{array}$ & $\begin{array}{c}90,4 \\
(88,2-92,6)\end{array}$ & $\begin{array}{c}93,8 \\
(91,2-96,4)\end{array}$ & $\begin{array}{c}94,2 \\
(91,4-97,1)\end{array}$ & $\begin{array}{c}91,9 \\
(85,3-98,6)\end{array}$ & \\
\hline Análise bruta & 1,00 & $\begin{array}{c}1,00 \\
(0,96-1,03)\end{array}$ & $\begin{array}{c}1,03 \\
(1,00-1,07)\end{array}$ & $\begin{array}{c}1,04 \\
(1,00-1,08)\end{array}$ & $\begin{array}{c}1,01 \\
(0,94-1,09)\end{array}$ & 0,214 \\
\hline Análise ajustada & 1,00 & $\begin{array}{c}1,00 \\
(0,97-1,04)\end{array}$ & $\begin{array}{c}1,04 \\
(1,00-1,08)\end{array}$ & $\begin{array}{c}1,04 \\
(1,00-1,09)\end{array}$ & $\begin{array}{c}1,03 \\
(0,95-1,11)\end{array}$ & 0,113 \\
\hline \multicolumn{7}{|c|}{$\begin{array}{l}\text { Recomendação para praticar atividade } \\
\text { física }\end{array}$} \\
\hline Prevalência & $\begin{array}{c}70,1 \\
(66,1-74,1)\end{array}$ & $\begin{array}{c}76,3 \\
(73,0-79,7)\end{array}$ & $\begin{array}{c}86,2 \\
(81,9-90,6)\end{array}$ & $\begin{array}{c}89,5 \\
(85,6-93,4)\end{array}$ & $\begin{array}{c}93,8 \\
(89,4-98,2)\end{array}$ & \\
\hline Análise bruta & 1,00 & $\begin{array}{c}1,09 \\
(1,02-1,17)\end{array}$ & $\begin{array}{c}1,23 \\
(1,14-1,32)\end{array}$ & $\begin{array}{c}1,28 \\
(1,19-1,37)\end{array}$ & $\begin{array}{c}1,34 \\
(1,24-1,44)\end{array}$ & $<0,001$ \\
\hline Análise ajustada & 1,00 & $\begin{array}{c}1,07 \\
(1,00-1,14)\end{array}$ & $\begin{array}{c}1,20 \\
(1,12-1,29)\end{array}$ & $\begin{array}{c}1,22 \\
(1,14-1,32)\end{array}$ & $\begin{array}{c}1,29 \\
(1,20-1,40)\end{array}$ & $<0,001$ \\
\hline \multicolumn{7}{|c|}{ Recomendação para não fumar } \\
\hline Prevalência & $\begin{array}{c}71,3 \\
(67,7-74,9)\end{array}$ & $\begin{array}{c}71,9 \\
(68,2-75,6)\end{array}$ & $\begin{array}{c}78,3 \\
(72,6-83,9)\end{array}$ & $\begin{array}{c}78,8 \\
(72,8-84,9)\end{array}$ & $\begin{array}{c}77,9 \\
(69,3-86,6)\end{array}$ & \\
\hline Análise bruta & 1,00 & $\begin{array}{c}1,01 \\
(0,94-1,08)\end{array}$ & $\begin{array}{c}1,10 \\
(1,01-1,19)\end{array}$ & $\begin{array}{c}1,11 \\
(1,01-1,21)\end{array}$ & $\begin{array}{c}1,09 \\
(0,97-1,23)\end{array}$ & 0,017 \\
\hline Análise ajustada & 1,00 & $\begin{array}{c}1,00 \\
(0,92-1,06)\end{array}$ & $\begin{array}{c}1,08 \\
(0,99-1,17)\end{array}$ & $\begin{array}{c}1,07 \\
(0,98-1,17)\end{array}$ & $\begin{array}{c}1,06 \\
(0,93-1,19)\end{array}$ & 0,099 \\
\hline \multicolumn{7}{|c|}{$\begin{array}{l}\text { Recomendação para não ingestão de } \\
\text { bebida alcoólica em excesso }\end{array}$} \\
\hline Prevalência & $\begin{array}{c}68,1 \\
(64,3-71,8)\end{array}$ & $\begin{array}{c}70,5 \\
(66,9-74,2)\end{array}$ & $\begin{array}{c}78,8 \\
(73,2-84,5)\end{array}$ & $\begin{array}{c}77,8 \\
(71,7-83,9)\end{array}$ & $\begin{array}{c}76,6 \\
(67,6-85,6)\end{array}$ & \\
\hline Análise bruta & 1,00 & $\begin{array}{c}1,04 \\
(0,96-1,11)\end{array}$ & $\begin{array}{c}1,16 \\
(1,06-1,27)\end{array}$ & $\begin{array}{c}1,14 \\
(1,04-1,26)\end{array}$ & $\begin{array}{c}1,13 \\
(0,99-1,28)\end{array}$ & 0,003 \\
\hline Análise ajustada & 1,00 & $\begin{array}{c}1,01 \\
(0,94-1,09)\end{array}$ & $\begin{array}{c}1,13 \\
(1,03-1,23)\end{array}$ & $\begin{array}{c}1,10 \\
(1,00-1,21)\end{array}$ & $\begin{array}{c}1,07 \\
(0,94-1,22)\end{array}$ & 0,039 \\
\hline \multicolumn{7}{|c|}{$\begin{array}{l}\text { Recomendação para fazer } \\
\text { acompanhamento regular }\end{array}$} \\
\hline Prevalência & $\begin{array}{c}84,3 \\
(81,4-87,1)\end{array}$ & $\begin{array}{c}87,2 \\
(84,7-89,6)\end{array}$ & $\begin{array}{c}90,4 \\
(86,4-94,4)\end{array}$ & $\begin{array}{c}92,7 \\
(89,7-95,8)\end{array}$ & $\begin{array}{c}94,3 \\
(90,9-97,6)\end{array}$ & \\
\hline Análise bruta & 1,00 & $\begin{array}{c}1,03 \\
(0,99-1,08)\end{array}$ & $\begin{array}{c}1,07 \\
(1,02-1,13)\end{array}$ & $\begin{array}{c}1,10 \\
(1,05-1,15)\end{array}$ & $\begin{array}{c}1,12 \\
(1,07-1,17)\end{array}$ & $<0,001$ \\
\hline Análise ajustada & 1,00 & $\begin{array}{c}1,03 \\
(0,99-1,08)\end{array}$ & $\begin{array}{c}1,07 \\
(1,01-1,13)\end{array}$ & $\begin{array}{c}1,10 \\
(1,04-1,15)\end{array}$ & $\begin{array}{c}1,11 \\
(1,05-1,17)\end{array}$ & $<0,001$ \\
\hline \multicolumn{7}{|c|}{ Solicitação de exame de sangue } \\
\hline Prevalência & $\begin{array}{c}80,7 \\
(77,5-83,9)\end{array}$ & $\begin{array}{c}84,1 \\
(80,6-87,5)\end{array}$ & $\begin{array}{c}84,0 \\
(78,4-89,5)\end{array}$ & $\begin{array}{c}89,9 \\
(86,1-93,6)\end{array}$ & $\begin{array}{c}86,8 \\
(75,2-98,3)\end{array}$ & \\
\hline Análise bruta & 1,00 & $\begin{array}{c}1,04 \\
(0,98-1,10)\end{array}$ & $\begin{array}{c}1,04 \\
(0,96-1,13)\end{array}$ & $\begin{array}{c}1,11 \\
(1,05-1,18)\end{array}$ & $\begin{array}{c}1,08 \\
(0,94-1,23)\end{array}$ & 0,043 \\
\hline Análise ajustada & 1,00 & $\begin{array}{c}1,04 \\
(0,98-1,09)\end{array}$ & $\begin{array}{c}1,03 \\
(0,95-1,12)\end{array}$ & $\begin{array}{c}1,11 \\
(1,05-1,18)\end{array}$ & $\begin{array}{c}1,07 \\
(0,94-1,21)\end{array}$ & 0,053 \\
\hline
\end{tabular}

(continua) 


\begin{tabular}{|c|c|c|c|c|c|c|}
\hline \multirow[t]{2}{*}{ Variáveis } & \multicolumn{5}{|c|}{ Escolaridade [\% (IC95\%)] } & \multirow[t]{2}{*}{ Valor de $p$ * } \\
\hline & Sem instrução & $\begin{array}{c}\text { Ensino } \\
\text { Fundamental } \\
\text { incompleto }\end{array}$ & $\begin{array}{c}\text { Ensino } \\
\text { Fundamental } \\
\text { completo/Médio } \\
\text { incompleto }\end{array}$ & $\begin{array}{l}\text { Ensino Médio } \\
\text { completo/ } \\
\text { Superior } \\
\text { incompleto }\end{array}$ & $\begin{array}{l}\text { Ensino } \\
\text { Superior } \\
\text { completo }\end{array}$ & \\
\hline \multicolumn{7}{|c|}{ Solicitação de exame de urina } \\
\hline Prevalência & $\begin{array}{c}68,6 \\
(64,5-72,6)\end{array}$ & $\begin{array}{c}75,7 \\
(72,3-79,0)\end{array}$ & $\begin{array}{c}77,4 \\
(71,0-83,8)\end{array}$ & $\begin{array}{c}77,5 \\
(71,0-84,0)\end{array}$ & $\begin{array}{c}77,0 \\
(65,0-89,0)\end{array}$ & \\
\hline Análise bruta & 1,00 & $\begin{array}{c}1,10 \\
(1,03-1,18)\end{array}$ & $\begin{array}{c}1,13 \\
(1,02-1,25)\end{array}$ & $\begin{array}{c}1,13 \\
(1,02-1,25)\end{array}$ & $\begin{array}{c}1,12 \\
(0,95-1,32)\end{array}$ & 0,037 \\
\hline Análise ajustada & 1,00 & $\begin{array}{c}1,10 \\
(1,03-1,18)\end{array}$ & $\begin{array}{c}1,13 \\
(1,01-1,25)\end{array}$ & $\begin{array}{c}1,13 \\
(1,02-1,25)\end{array}$ & $\begin{array}{c}1,12 \\
(0,95-1,31)\end{array}$ & 0,043 \\
\hline \multicolumn{7}{|c|}{ Solicitação de eletrocardiograma } \\
\hline Prevalência & $\begin{array}{c}62,7 \\
(58,7-66,7)\end{array}$ & $\begin{array}{c}71,5 \\
(67,7-75,2)\end{array}$ & $\begin{array}{c}72,2 \\
(65,2-79,3)\end{array}$ & $\begin{array}{c}84,3 \\
(79,8-88,8)\end{array}$ & $\begin{array}{c}80,9 \\
(69,7-92,0)\end{array}$ & \\
\hline Análise bruta & 1,00 & $\begin{array}{c}1,14 \\
(1,05-1,24)\end{array}$ & $\begin{array}{c}1,15 \\
(1,03-1,30)\end{array}$ & $\begin{array}{c}1,35 \\
(1,24-1,46)\end{array}$ & $\begin{array}{c}1,29 \\
(1,11-1,50)\end{array}$ & $<0,001$ \\
\hline Análise ajustada & 1,00 & $\begin{array}{c}1,12 \\
(1,03-1,22)\end{array}$ & $\begin{array}{c}1,12 \\
(1,00-1,26)\end{array}$ & $\begin{array}{c}1,32 \\
(1,21-1,43)\end{array}$ & $\begin{array}{c}1,26 \\
(1,09-1,45)\end{array}$ & $<0,001$ \\
\hline \multicolumn{7}{|c|}{ Solicitação de teste de esforço } \\
\hline Prevalência & $\begin{array}{c}25,6 \\
(22,0-29,2)\end{array}$ & $\begin{array}{c}36,1 \\
(31,9-40,4)\end{array}$ & $\begin{array}{c}43,2 \\
(35,8-50,6)\end{array}$ & $\begin{array}{c}51,0 \\
(43,8-58,2)\end{array}$ & $\begin{array}{c}62,5 \\
(51,2-73,7)\end{array}$ & \\
\hline Análise bruta & 1,00 & $\begin{array}{c}1,41 \\
(1,18-1,69)\end{array}$ & $\begin{array}{c}1,69 \\
(1,35-2,12)\end{array}$ & $\begin{array}{c}1,99 \\
(1,63-2,43)\end{array}$ & $\begin{array}{c}2,44 \\
(1,95-3,06)\end{array}$ & $<0,001$ \\
\hline Análise ajustada & 1,00 & $\begin{array}{c}1,35 \\
(1,13-1,62)\end{array}$ & $\begin{array}{c}1,59 \\
(1,26-2,00)\end{array}$ & $\begin{array}{c}1,87 \\
(1,52-2,30)\end{array}$ & $\begin{array}{c}2,26 \\
(1,80-2,83)\end{array}$ & $<0,001$ \\
\hline \multicolumn{7}{|c|}{ Todos os indicadores ** } \\
\hline Prevalência & $\begin{array}{c}16,9 \\
(13,8-20,1)\end{array}$ & $\begin{array}{c}22,7 \\
(19,2-26,2)\end{array}$ & $\begin{array}{c}31,7 \\
(24,6-38,7)\end{array}$ & $\begin{array}{c}34,2 \\
(27,4-41,0)\end{array}$ & $\begin{array}{c}40,0 \\
(31,1-49,0)\end{array}$ & \\
\hline Análise bruta & 1,00 & $\begin{array}{c}1,34 \\
(1,06-1,70)\end{array}$ & $\begin{array}{c}1,87 \\
(1,39-2,50)\end{array}$ & $\begin{array}{c}2,02 \\
(1,54-2,64)\end{array}$ & $\begin{array}{c}2,36 \\
(1,77-3,16)\end{array}$ & $<0,001$ \\
\hline Análise ajustada & 1,00 & $\begin{array}{c}1,28 \\
(1,00-1,62)\end{array}$ & $\begin{array}{c}1,75 \\
(1,30-2,36)\end{array}$ & $\begin{array}{c}1,87 \\
(1,42-2,47)\end{array}$ & $\begin{array}{c}2,14 \\
(1,59-2,86)\end{array}$ & $<0,001$ \\
\hline
\end{tabular}

IC95\%: intervalo de 95\% de confiança.

* Teste de Wald para tendência linear análise ajustada para sexo, idade, cor da pele, situação conjugal e região de residência;

** Idosos com hipertensão que receberam todos os indicadores avaliados: recomendação para alimentação saudável, manter o peso adequado, ingerir menos sal, prática de atividade física, não fumar, não ingestão de bebida alcoólica em excesso e fazer acompanhamento regular/solicitação de exame de sangue, urina, eletrocardiograma e teste de esforço.

\section{Discussão}

Os achados deste estudo apontaram que desigualdades na atenção aos idosos com hipertensão ainda persistem, já que idosos mais escolarizados tiveram maior prevalência dos indicadores de atenção oferecida por profissional de saúde quando comparados aos idosos sem instrução. Entre o recebimento de todos os indicadores, encontrou-se o mesmo padrão, sugerindo que, apesar da diminuição das iniquidades no acesso aos serviços de saúde no Brasil 6, a atenção oferecida apresenta importantes disparidades 10 .

Aproximadamente três quartos da amostra estudada não recebeu todos os indicadores de atenção avaliados, o que ao extrapolar para a população-alvo, representa cerca de oito milhões de idosos brasileiros portadores de HAS. Esse número absoluto é importante para dimensionar a falta de recebimento de ações de prevenção e promoção de saúde, que são estratégias fundamentais para diminuir as complicações e agravos gerados pela falta de cuidados específicos aos indivíduos acometidos pela doença 1 . 
Ao analisar os indicadores separadamente, nota-se que as recomendações foram mais prevalentes do que a solicitação de exames, no entanto ainda não atingem a totalidade da população-alvo. Flores et al. 18 evidenciaram menores prevalências de recomendações para hábitos saudáveis, todavia se tratava de uma amostra de idosos saudáveis e doentes. É possível que os profissionais de saúde estejam perdendo a oportunidade de dispensação de ações de promoção da saúde 19 , focalizando as recomendações nos indivíduos que já possuem alguma doença. Contudo, a comparabilidade é uma dificuldade presente, tendo em vista a escassez de estudos que investiguem os mesmos indicadores avaliados em idosos portadores de hipertensão.

Além disso, o fato de a solicitação de exames, mesmo sendo uma medida básica para o controle da HAS, estar sendo menos realizada pelos profissionais de saúde pode ser em função de que tal ação é mais centrada no médico, já as recomendações são estratégias multiprofissionais que envolvem ações exercidas não somente pelo médico, mas que deveriam ser realizadas por todos os profissionais de saúde 19,20 .

Assim, as recomendações sobre hábitos saudáveis, consideradas ações de baixo custo, ainda não estão adequadas, e deveriam ser realizadas em todo contato dos profissionais de saúde com os indivíduos, especialmente no momento da consulta 5,19. Estudos 13,21 mostram que a adoção de um estilo de vida saudável tem efeito na redução da pressão arterial, ajudam no controle de fatores de risco para outros agravos, aumentam a eficácia do tratamento medicamentoso e diminui o risco cardiovascular, reduzindo os gastos do sistema de saúde com medicações e serviços especializados 1 .

A redução do consumo de sal é uma das principais estratégias de prevenção e controle da pressão arterial ${ }^{20}$. No presente estudo, a recomendação para ingerir menos sal não apresentou diferença entre os grupos de escolaridade, todavia outras recomendações como alimentação saudável, manter o peso adequado, praticar atividade física, não ingerir bebida alcoólica em excesso e fazer acompanhamento regular se distribuíram de maneira desigual entre os grupos de escolaridade. Esse resultado pode sugerir um problema, pois a HAS é uma doença multicausal cujo manejo e controle depende de um conjunto de fatores, e que a mudança de estilo de vida direcionada a uma condição isolada pode não resultar na diminuição dos riscos de complicações e manejo adequado da doença.

As ações de promoção de saúde realizadas nos serviços de saúde devem auxiliar na melhoria da qualidade de vida dos indivíduos configurando em maior qualidade da atenção 2 , porém é necessário que os profissionais de saúde estejam engajados no processo educativo, realizando as recomendações de forma que esses indivíduos compreendam o processo, além de incentivar o autocuidado 22 . Um estudo realizado nos Estados Unidos que avaliou a qualidade do cuidado a pessoas com hipertensão evidenciou que 57\% dos indivíduos receberam cuidado considerado "ótimo", apresentando maior chance de ter a pressão arterial controlada. Além disso, os indivíduos com 50 anos ou mais e com outras comorbidades apresentaram maior chance de receber um cuidado "ótimo" 12. Outro estudo, também nos Estados Unidos, mostrou que idosos que receberam recomendações de profissionais de saúde para o manejo da HAS tiveram maior engajamento em um estilo de vida saudável 13 .

A escolaridade foi utilizada como proxy de nível socioeconômico, mas também reflete o nível de conhecimento do indivíduo, sendo possível que idosos mais escolarizados compreendam melhor as recomendações realizadas e a importância das solicitações de exames pelos profissionais de saúde 23,24 . O uso de apenas uma variável para as análises pode representar uma limitação do estudo, entretanto a escolaridade também é utilizada como proxy para estudar desigualdades em saúde 9 e apresenta resultados semelhantes ao evidenciado por indicadores de renda 10 .

Em relação aos possíveis vieses, cabe ressaltar o viés de recordatório, já que os idosos podem apresentar certa dificuldade em lembrar eventos passados. Entretanto, por serem idosos com hipertensão, é provável que sua frequência aos serviços de saúde seja maior do que a população em geral, diminuindo, em média, o tempo decorrido entre a última consulta e a entrevista, podendo minimizar o efeito do viés.

Estudos com essa população, que avaliam a atenção prestada aos usuários nos serviços de saúde, realizam inquéritos domiciliares utilizando esse tipo de metodologia 13,25. Ainda, o viés de sobrevivência pode estar presente por se tratar de um delineamento transversal que mede em um único ponto; é possível que os idosos com hipertensão e menores condições socioeconômicas, nesse caso escolaridade, não estivessem vivos no momento da coleta, fazendo que as prevalências dos indicadores de atenção oferecida por profissional de saúde esteja superestimada, bem como sua associação 
com a exposição. Tal reflexão pode sugerir que a situação da atenção oferecida é ainda pior do que a encontrada, tornando necessária a realização de mais estudos que aprofundem essa temática.

Em conclusão, mesmo com os avanços no acesso aos serviços de saúde e nas estratégias voltadas aos indivíduos portadores de DCNT 1,4, a atenção oferecida por profissional de saúde está deficiente em todo país, visto que menos de um quarto dos idosos com hipertensão recebeu todos os indicadores avaliados. Além disso, as desigualdades ainda representam um problema marcante no país, evidenciado pela diferença no recebimento dos indicadores de acordo com a escolaridade, em que os mais escolarizados apresentaram melhores prevalências. Sendo assim, há necessidade de políticas públicas que objetivem reduzir esse problema, com ações que sejam importantes para um bom manejo da HAS, como a promoção da saúde e o autocuidado. Com as implicações do aumento da população idosa e das DCNT, esforços para melhorar a atenção oferecida pelos profissionais de saúde serão fundamentais para superar esse desafio em todo o território nacional.

\section{Colaboradores}

R. G. Neves realizou análise de dados, revisão de literatura e redação do manuscrito. S. M. S. Duro colaborou na redação do texto e revisão da análise de dados. T. R. Flores colaborou na redação do manuscrito e análise de dados. C. S. Costa colaborou na revisão do manuscrito e análise de dados. A. Wendt colaborou na revisão do texto e revisão da análise de dados. B. P. Nunes colaborou na revisão, redação e análise de dados. F. C. Wehrmeister colaborou na revisão do texto e análise de dados. E. Tomasi colaborou na redação do manuscrito, revisão da literatura e da análise de dados.

\section{Referências}

1. Schmidt M, Duncan B, Silva G, Menezes A, Monteiro C, Barreto S. Doenças crônicas não transmissíveis no Brasil: carga e desafios atuais. Lancet 2011; 377:1949-61.

2. Departamento de Atenção Básica, Secretaria de Atenção à Saúde, Ministério da Saúde. Envelhecimento e saúde da pessoa idosa. Brasília: Ministério da Saúde; 2007. (Caderno de Atenção Básica, 19) (Série A. Normas e Manuais Técnicos).

3. Azevedo Barros MB, Francisco PMSB, Zanchetta LM, César CLG. Tendências das desigualdades sociais e demográficas na prevalência de doenças crônicas no Brasil, PNAD: 2003-2008. Ciênc Saúde Coletiva 2011; 16:3755-68.

4. Duncan BB, Chor D, Aquino EML, Benseñor IJM, Mill JG, Schmidt MI, et al. Doenças crônicas não transmissíveis no Brasil: prioridade para enfrentamento e investigação. Rev Saúde Pública 2012; 46 Suppl 1:S126-34.

5. Departamento de Atenção Básica, Secretaria de Atenção à Saúde, Ministério da Saúde. Estratégias para o cuidado da pessoa com doença crônica. Brasília: Ministério da Saúde; 2014. (Caderno de Atenção Básica, 35).

6. Macinko J, Lima-Costa MF. Horizontal equity in health care utilization in Brazil, 1998-2008. Int J Equity Health 2012; 11:33.

7. Macinko J, Harris MJ. Brazil's family health strategy: delivering community-based primary care in a universal health system. N Engl J Med 2015; 372:2177-81.

8. Marmot M. Social determinants of health inequalities. Lancet 2005; 365:1099-104. 
9. Galobardes B, Shaw M, Lawlor DA, Lynch JW, Smith GD. Indicators of socioeconomic position (part 1). J Epidemiol Community Health 2006; 60:7-12.

10. Nunes BP, Tomasi E, Duro SMS, Facchini LA. Desigualdades socioeconômicas no acesso e qualidade da atenção nos serviços de saúde. Rev Saúde Pública 2014; 48:968-76.

11. Viana SM, Nunes A, Santos J, Barata R. Medindo as desigualdades em saúde no Brasil: uma proposta de monitoramento. Brasília: Organização Pan-Americana da Saúde; 2001.

12. Asch SM, McGlynn EA, Hiatt L, Adams J, Hicks J, DeCristofaro A, et al. Quality of care for hypertension in the United States. BMC Cardiovascular Disorders 2005; 5:1.

13. Walker RL, Gee ME, Bancej C, Nolan RP, Kaczorowski J, Joffres M, et al. Health behaviour advice from health professionals to Canadian adults with hypertension: results from a national survey. Can J Cardiol 2011; 27:446-54.

14. Souza-Júnior PRB, Freitas MPS, Antonaci GA, Szwarcwald CL. Desenho da amostra da Pesquisa Nacional de Saúde 2013. Epidemiol Serv Saúde 2015; 24:207-16.

15. Maria DI. O movimento pela qualidade nos serviços de saúde e enfermagem. Rev Bras Enferm 2006; 59:84-8.

16. Donabedian A. The quality of care: How can it be assessed? JAMA 1988; 260:1743-8.

17. Barros AJ, Hirakata VN. Alternatives for logistic regression in cross-sectional studies: an empirical comparison of models that directly estimate the prevalence ratio. BMC Med Res Methodol 2003; 3:21.

18. Flores TR, Nunes BP, Assunção MCF, Bertoldi AD. Hábitos saudáveis: que tipo de orientação a população idosa está recebendo dos profissionais de saúde? Rev Bras Epidemiol 2016; 19:167-80.
19. Silva S, Facchini L, Tomasi E, Piccini R, Thumé E, Silveira D. Recebimento de orientação sobre consumo de sal, açúcar e gorduras em adultos: um estudo de base nacional. Rev Bras Epidemiol 2013; 16:995-1004.

20. Departamento de Atenção Básica, Secretaria de Atenção à Saúde, Ministério da Saúde. Estratégias para o cuidado da pessoa com doença crônica. Hipertensão arterial sistêmica. Brasília: Ministério da Saúde; 2013.

21. Sega R, Facchetti R, Bombelli M, Cesana G, Corrao G, Grassi G, et al. Prognostic value of ambulatory and home blood pressures compared with office blood pressure in the general population follow-up results from the Pressioni Arteriose Monitorate e Loro Associazioni (PAMELA) Study. Circulation 2005; 111: 1777-83.

22. Mendes EV. O cuidado das condições crônicas na atenção primária à saúde: o imperativo da consolidação da estratégia da saúde da família. Brasília: Organização Pan-Americana da Saúde; 2012.

23. Lopes ACS, Toledo MTT, Câmara AMCS, Menzel H-JK, Santos LC. Condições de saúde e aconselhamento sobre alimentação e atividade física na Atenção Primária à Saúde de Belo Horizonte-MG. Epidemiol Serv Saúde 2014; 23:475-86

24. Sturmer G, Dias-da-Costa JS, Olinto MTA, Menezes AMB, Gigante DP, Macedo S. O manejo não medicamentoso da hipertensão arterial sistêmica no Sul do Brasil. Cad Saúde Pública 2006; 22:1727-37.

25. Lima-Costa MF, Loyola Filho AI, Matos DL. Tendências nas condições de saúde e uso de serviços de saúde entre idosos brasileiros: um estudo baseado na Pesquisa Nacional por Amostra de Domicílios (1998, 2003). Cad Saúde Pública 2007; 23:2467-78. 


\section{Abstract}

This study aimed to assess the attention paid by health professionals to elderly individuals with hypertension and related inequalities in Brazil, using level of schooling as the socioeconomic indicator. This was a cross-sectional study with data from the Brazilian National Health Survey, 2013. Attention was assessed according to the following indicators: recommendations for healthy eating, maintaining adequate weight, less salt, regular exercise, prevention of smoking and excessive drinking, and regular medical checkups with blood and urine tests, ECG, and stress test. The recommendations to consume less salt (91\%), healthy diet (88\%), and regular medical checkups (88\%) were the most common. Elderly with complete university degrees showed the highest prevalence rates for recommendations to maintain a healthy diet $(P R=1.12)$, adequate weight $(P R=1.21)$, regular exercise $(P R=1.29)$, regular medical checkups $(P R=1.11)$, and stress test $(P R=2.26)$ and all the indicators combined $(P R=2.14)$ when compared to elderly without schooling. The attention paid to the elderly by health professionals is suboptimal throughout the country. In addition, inequalities in attention to the elderly's needs still persist. Given the growth in the elderly population and chronic non-communicable diseases, efforts are needed to improve the quality of their care and mitigate the related inequalities.

Chronic Disease; Hypertension; Quality of Health Care; Aged

\section{Resumen}

El objetivo fue evaluar la atención ofrecida por los profesionales de salud a los ancianos con hipertensión y las desigualdades asociadas, utilizando la escolaridad como indicador socioeconómico, en Brasil. Estudio transversal, con datos de la Encuesta Nacional de Salud, en 2013. La atención ofrecida fue evaluada mediante indicadores: recomendaciones para alimentación saludable, mantener el peso adecuado, ingerir menos sal, practicar actividad física, no fumar, no ingerir bebida alcohólica en exceso, realizar un seguimiento regular y solicitar exámenes de sangre y orina, electrocardiograma y test de esfuerzo. Las recomendaciones para ingerir menos sal (91\%), alimentación saludable (88\%) y realizar un seguimiento regular (88\%) fueron las más prevalentes. Los ancianos con la enseñanza superior completada presentaron mayores prevalencias de recomendaciones para mantener una alimentación saludable $(R P=1,12)$, mantener el peso adecuado $(R P=1,21)$, practicar actividad física $(R P=1,29)$, seguimiento regular $(R P=1,11)$, solicitar el test de esfuerzo $(R P=2,26)$ y la recepción de todos los indicadores $(R P=2,14)$, en relación con los ancianos sin instrucción. La atención ofrecida por un profesional de salud es deficiente en todo el país. Asimismo, las desigualdades todavía persisten. Teniendo en vista el aumento de la población anciana y de las enfermedades crónicas no transmisibles, se necesitan esfuerzos para mejorar la calidad de la atención y disminuir las desigualdades.

Doença Crônica; Hipertensión; Calidad de la Atención de Salud; Anciano
Recebido em 15/Dez/2015

Versão final reapresentada em 12/Set/2016

Aprovado em 22/Set/2016 